\title{
Producción científica en el campo de los estudios de género: análisis de revistas seleccionadas de Web of Science (2008-2018)
}

\author{
Juan Pablo Sokil*, Laura Osorio* \\ *Observatorio Iberoamericano de la Ciencia, la Tecnología y la Sociedad. \\ Organización de Estados Iberoamericanos. (OCTS-OEI) \\ Correo-e: juanpablosokil@gmail.com | ORCID iD: http://orcid.org/0000-0003-4700-2145 \\ Correo-e: losorio.oei@gmail.com | ORCID iD: https://orcid.org/0000-0001-7072-3851
}

Recibido: 08-10-20; 2a versión: 26-01-21; Aceptado: 02-03-21; Publicado: 31-01-2022

Cómo citar este artículo/Citation: Sokil, J. P.; Osorio, L. (2022). Producción científica en el campo de los estudios de género: análisis de revistas seleccionadas de Web Of Science (2008-2018). Revista Española de Documentación Científica, 45 (1), e3120. https://doi.org/ 10.3989/redc.2022.1.1849

Resumen: La siguiente investigación analiza las publicaciones científicas de estudios de género a nivel mundial en revistas seleccionadas indexadas en Web of Science (WOS) entre 2008 y 2018. Los objetivos son indagar sobre la dinámica de participación de las y los autores dentro de esta área de conocimiento y que tópicos investigan, por separado y en conjunto. Para esto, se utilizan algoritmos de clasificación de sexo y de modelado de tópicos. Los resultados muestran que los estudios de género son una de las áreas de investigación más feminizadas y por ende con mayor brecha de género, además destacan que no hubo cambios relevantes dentro del periodo analizado. Los tópicos identificados permiten segmentar los tipos de autoría: ellas se especializan en feminismo, política, violencia, entre otros, ellos no tienen ninguna especialización y la autoría en conjunto lo hace en áreas asociadas a la medicina/salud y estadística/metodología.

Palabras clave: estudios de género; brecha de género; bibliometría; modelado de tópicos; predicción de sexo

\section{Scientific production in the field of gender studies: an alysis of se lected Web of Science journals (2008-2018)}

Abstract: The following research analyzes the scientific production of gender studies worldwide indexed in the Web of Science (WOS) between 2008 and 2018. The objectives are to analyze the dynamics of participation of women and men within this area and investigate what topics they investigate (separately and together) using sex classification and topic modeling algorithms. The results show that gender studies are one of the most feminized research areas, with the greatest gender gap and that there were no changes throughout the selected period. The identified topics segment the preferences between the authors: female authors specialize in feminism, politics, violence, male authors have a distributed production and mixed authorship specializes in medicine/ health and statistics/ methodology.

Keywords: gender studies; gender gap; bibliometrics; topic modeling; sex prediction

Copyright: (c) 2022 CSIC. Este es un artículo de acceso abierto distribuido bajo los términos de la licencia de uso y distribución Creative Commons Reconocimiento 4.0 Internacional (CC BY 4.0). 


\section{INTRODUCCIÓN}

La publicación científica de artículos en revistas académicas es una de las actividades fundamentales no sólo para contribuir al avance de las fronteras del conocimiento sino también a la toma de decisión política. Dicha actividad responde a fenómenos estructurales que inciden en qué temas publicar, cómo llevarlo a cabo y quiénes participan en este proceso de publicación. Con respecto a esto último, es sabido que existe una disparidad entre las y los autores, lo que se evidencia no sólo en su participación en el campo científico y tecnológico sino también en su producción y temas de trabajo.

Los estudios de género son uno de los tantos campos de investigación científica que, en particular, remontan su origen al movimiento feminista de la década del 70 (Amorós y de Miguel Álvarez, 2005; González y Fernández Jimeno, 2016; Núñez Noriega, 2016). En la actualidad incluyen un conjunto heterogéneo de investigaciones que poseen al género como categoría central: estudios sobre feminismo, estudios de la mujer, estudios del hombre y los estudios de diversidad sexual y abarcan incontables temáticas de estudio, entre ellas, trabajo, salud, violencia, educación o derechos (Morales, 2018).

Teniendo en cuenta lo anterior, la siguiente investigación analiza la producción científica de estudios de género a nivel mundial en revistas seleccionadas indexadas en Web of Science (WOS) entre 2008 y 2018 . Se busca dar cuenta de la dinámica de participación de las y los autores dentro de esta área de conocimiento, así como indagar qué tópicos investigan las autoras y los autores (por separado y en conjunto), sí coinciden o difieren y cómo opera la autoría conjunta dentro de este campo de conocimiento.

\section{ANTECEDENTES}

La brecha de género dentro de la producción científica es un fenómeno ampliamente estudiado (López-Bassols y otros, 2018; Albornoz y otros, 2018; UNESCO, 2017 y 2019). Existen desarrollos teóricos que analizan en detalle las causas de dicha disparidad y desentrañan aquellos fenómenos estructurales y prácticas sociales y culturales que inciden en que la participación de las mujeres no se lleve a cabo de la misma manera que los hombres.

Si bien las consecuencias de esta brecha abarcan infinidad de situaciones, empíricamente (dentro de las publicaciones científicas) pueden observarse en dos hechos puntuales: La menor productividad que tienen las mujeres en todas las disciplinas científicas, sobre todo en los programas de ciencia, tecnología, ingeniería y matemática (STEM por sus siglas en inglés) y la feminización/masculinización de ciertas áreas del conocimiento.

Con respecto al primer punto, en numerosos estudios se observa que las mujeres no solo publican menos que los hombres, sino que también reciben menos citas y por ende obtienen menor reconocimiento (Zuckerman y otros, 1991; Flores Espinola, 2010). Entre los factores causantes se pueden enumerar varios: la relación de colaboración con sus mentores y colegas, el sexismo presente en todas las facetas previas a la publicación en revistas, la maternidad y el cuidado del hogar (Long, 1990 en Flores Espinola, 2010).

En lo referente al segundo punto, se observa que la distribución de investigadores según áreas es muy distinta; en las de tipo STEM hay poca presencia de mujeres mientras que en áreas asociadas al cuidado y educación existe una sobre representación de investigadoras (Penner, 2015; Elsevier, 2015; Elsevier, 2017; Elsevier, 2020; Thelwall y otros, 2019; Albornoz y otros, 2018). Estos sesgos en la participación en disciplinas científicas se encuentran fuertemente asociados a cuestiones culturales y a la construcción y perpetuación de estereotipos vinculados a los sexos (González y Fernández Jimeno, 2016; López-Bassols y otros, 2018; Papadópulos y Radakovich, 2006, Thelwall y otros, 2019).

Si bien este tipo de estudios se han extendido a muchos campos del conocimiento, su presencia dentro de la producción científica sobre estudios de la mujer y el género ha sido casi nula. Esto resulta contradictorio, ya que la aparición de estos estudios fue el punto de partida para el análisis de las desigualdades de las mujeres en todos los campos y esferas de la vida social, incluido el ámbito científico y tecnológico (Bonder, 1984 y 2004; Pérez Sedeño, 2001; González y Fernández Jimeno, 2016; Maffia 2008; Flores Espinola, 2010 y 2016).

Entre las investigaciones que abarcan esta área se encuentra la realizada por Thelwall y otros (2019), donde se analiza la distribución de las y los autores dentro de todas las categorías y subcategorías temáticas propuestas por SCOPUS (un total de 255), entre ellas los estudios de género, siendo ésta la segunda con mayor presencia de mujeres, $64 \%$, después de enfermería que cuenta con un $67 \%$. Cabe aclarar que este análisis se realiza solamente para las publicaciones cuyo primer autor o autora sea de Estados Unidos (que representa alrededor del $20 \%$ de la producción científica de SCOPUS). Asimismo, la investigación realizada por Flores Espinola (2016), realiza un recorte a nivel de revistas más no de país, donde la autora selec- 
ciona un conjunto de tres revistas especializadas en género y analiza con mayor detalle la autoría de las publicaciones. Si bien, la muestra es pequeña, da cuenta que alrededor del $65 \%$ son autoras.

Además de la distribución porcentual de autoras y autores, existen estudios recientes que se enfocan en los diferentes temas que se investigan. Desde su creación, los estudios de la mujer y luego los estudios de género han tenido una tendencia a profundizar en fenómenos tales como el acceso de las mujeres a la educación, los patrones sexistas en las experiencias escolares, el mercado de trabajo y factores de discriminación, salud (sexual y reproductiva), participación en la política, lugares de decisión, pobreza, condiciones de vida, violencia hacia la mujer, prevención y reparación, entre otras (Bonder, 2004).

En particular, dentro de los estudios bibliométricos aparecen aquéllos que presentan mapas temáticos de las investigaciones sobre género (a partir de las palabras claves), como ocurre en Elsevier (2015; 2017). En ambos casos, los mapas dan cuenta de dos grandes áreas temáticas: la biomedicina y las ciencias sociales. El análisis más detallado muestra que los hombres se encuentran asociados a palabras como metodología, probabilidad y modelos teóricos, mientras las mujeres lo hacen a términos como familia, hijos, mujeres, embarazo. A un nivel más general, Thelwall y otros (2019) estudian la probabilidad de aparición de ciertas palabras según el sexo de las y los autores, concluyendo que el término women (mujer) es la palabra más asociada a las autoras mientras que techniques (técnicas) la más asociada a los hombres. En Flores Espinola (2016), se concluye que la biomedicina es el área más estudiada en las revistas de género.

Teniendo en cuenta lo anterior, nuestro objetivo retoma y sintetiza las propuestas arriba expuestas con el fin de analizar la dinámica de participación de las y los autores dentro del universo seleccionado. Adicionalmente, observar cómo esta dinámica de producción ha evolucionado en los últimos años y si ha habido algún cambio. También estudiar si las temáticas dentro de los estudios de género se asocian a alguno de los sexos en particular y de ser cierto, si producen resultados similares a los obtenidos en Elsevier $(2015 ; 2017 ; 2020)$ o Thelwall y otros (2019).

Para guiar la investigación, se responderá a una serie de interrogantes: ¿Cuál es la participación de las y los autores dentro de los estudios de género?, ¿Ha habido algún cambio en los últimos años?, ¿Qué temas investigan las autoras?, ¿Son los mismos que los autores?, cuando investigan por separado, ¿lo hacen en temas distintos?

\section{DATOS Y MÉTODOS}

La recolección de datos fue realizada a través de Web of Science (WOS) para el periodo 2008-2018. Este servicio de información científica provee acceso a bases de datos bibliométricas, su colección incluye Science Citation Index Expanded (SCI-Expanded), Social Sciences Citation Index (SSCI), Arts \& Humanities Citation Index (A\&HCI) y Emerging Sources Citation index (ESCI), esta última a partir del año $2015^{1}$.

Con el propósito de definir el universo de análisis se tomaron dos criterios: 1) aquellas publicaciones que pertenezcan a una revista sobre estudios de la mujer, 2) aquellas publicaciones que pertenezcan al área de ciencias sociales y que además incluyan la palabra género en su título, resumen o palabra clave.' Web of Science (WOS) define 5 grandes áreas de la ciencia (entre las que se encuentra ciencias sociales), que a su vez se dividen en 250 disciplinas (una de ellas es estudios de la mujer) ${ }^{2}$.

Ambos criterios pueden ser resumidos mediante la siguiente estrategia de búsqueda: (TS='gender' AND SU='Social Sciences') or WC="Women's Studies".

Asimismo, se descargaron todos los archivos en formato txt, se unificaron y procesaron mediante el software $\mathrm{R}$ y se utilizaron 5 variables: UT (Código identificador), TI (Título), AB (Resumen), PY (Año de publicación) y AF (Nombre y Apellido de autores).

Seguidamente, se extrajeron todos los nombres de pila del campo AF y mediante un algoritmo de clasificación de sexo se calculó la probabilidad que tenía cada nombre de ser hombre o mujer (Mullen, 2018). Cabe aclarar que este tipo de algoritmos se basan en registros históricos de nacimientos y asocian los nombres a un determinado sexo biológico, por ello se habla de sexo en lugar de género. Siguiendo lo realizado en Elsevier $(2015 ; 2017)$ se descartaron los casos en los que no fue posible identificar el sexo $^{3}$.

El universo de análisis constó de 21.309 publicaciones y 40.376 autoras y autores distintos distribuidos entre los años 2008 y 2018.

Se construyeron una serie de indicadores:

1. Distribución de autores por sexo (cantidad de autoras y autores sobre el total de las y los autores identificados).

2. Cociente entre autoras y autores (relación entre el total de las y los autores identificados).

3. Tipo de autoría (características de colaboración entre las y los autores de la publicación dividi- 
da en cinco categorías excluyentes: única autora, único autor, múltiples autoras, múltiples autores y autoría mixta - participan al menos un hombre y una mujer-).

4. Participación en publicaciones por sexo (cantidad de publicaciones donde participa al menos una autora, o un autor, sobre el total de publicaciones).

5. Publicaciones por sexo (cantidad de artículos firmados por las y los autores).

Para identificar los tópicos dentro de las publicaciones se utilizó Asignación Latente de Dirichlet - (LDA por sus siglas en inglés) (Blei y otros. 2003), un algoritmo de modelado de tópicos que procesa el contenido de los documentos (en este caso los resúmenes $[A B]$ y títulos [TI]), extrae $\mathrm{n}$-gramas (conjuntos de 1 a $\mathrm{n}$ palabras - en este caso 4), identifica aquéllos con alta probabilidad de aparecer juntos y los agrupa en clústeres o tópicos, asume que cada tópico es una distribución de n-gramas (o palabras), y que cada documento se compone de una distribución de palabras y una proporción de tópicos.

Para llevar a cabo el proceso se definieron los parámetros Alfa y Beta: en el primero, a mayor vaIor, cada documento estará representando por más tópicos; en el segundo, a mayor valor, cada tópico estará representado por más n-gramas (palabras). En este caso, los parámetros utilizados fueron los propuestos en Vogel y Jurafsky (2012): números de tópicos: 100, Alfa asimétrico y Beta igual al total de n-gramas distintos sobre la cantidad de tópicos. El algoritmo brinda una serie de parámetros adicionales, entre ellos Theta (distribución de tópicos por documento, es decir, cuánto de cada tópico tiene cada documento); este valor permitió calcular la distribución porcentual de tópicos, a nivel total y por tipo de coautoría.

Con estas mismas distribuciones se construyó el indicador puntaje de tópicos (división entre la distribución porcentual de tópicos de cada tipo de coautoría y la distribución porcentual de tópicos total), que permite identificar en qué tópico se es- pecializa (y no se especializa) cada segmento, tomando como umbral un 0,25 mayor o menor con respecto al promedio total.

\section{RESULTADOS}

\subsection{Características de las investigaciones}

Dentro de las 21.309 publicaciones analizadas que cumplieron con los criterios metodológicos definidos anteriormente, se encontraron $27.753 \mathrm{mu}$ jeres y 12.623 hombres, es decir que el $68,7 \%$ del total de las y los autores fueron mujeres. El cociente entre las y los autores muestra que existieron 2,19 mujeres publicando por cada hombre.

La tabla I resume la distribución según el tipo de autoría y muestra que un $32,5 \%$ de las publicaciones fueron realizadas por una única autora, ese porcentaje aumentó a 55,9\% cuando se habla de artículos con autoría exclusivamente de mujeres (sin importar la cantidad). Asimismo, hubo un $85,6 \%$ de publicaciones donde participó al menos una autora. Con respecto a los autores, se observaron porcentajes mucho más bajos, apenas un $9,6 \%$ de las publicaciones fueron realizadas por un autor y un $14,4 \%$ fueron con autoría de hombres exclusivamente (sin importar la cantidad) y el total de publicaciones donde participó al menos un autor fue de $44,1 \%$.

Otra alternativa para analizar el tipo de autoría es a nivel sexo, es decir cómo se componen las publicaciones que tienen al menos una autora y como lo hacen aquellas que tienen al menos un autor, esa información se presenta en el gráfico 1.

En el caso de los documentos con alguna mujer (a la izquierda), predominan las publicaciones con una única autora $(37,9 \%)$, mientras que en los documentos con algún hombre (a la derecha), destaca la autoría mixta $(67,4 \%)$, de hecho, este valor casi duplica al que tienen las autoras $(34,7 \%)$. Ambos segmentos presentan una distribución muy heterogénea, y se observa que los autores tienen una dependencia mucho más fuerte de autoras al momento de publicar.

Tabla I. Distribución por tipo de autoría de publicaciones en estudios de género en revistas indexadas en WOS

\begin{tabular}{|l|c|c|c|}
\hline Tipo de Autoría & Publicaciones & Porcentaje & Porcentaje Acum \\
\hline Única autora & 6.929 & $32,5 \%$ & $55,9 \%$ \\
\hline Múltiples autoras & 4.982 & $23,4 \%$ & $29,7 \%$ \\
\hline Autoría mixta & 6.336 & $29,7 \%$ & $14,4 \%$ \\
\hline Múltiples autores & 1.017 & $4,8 \%$ & $100,0 \%$ \\
\hline Único autor & 2.045 & $9,6 \%$ & $100,0 \%$ \\
\hline Total & 21.309 & & \multirow{2}{*}{10} \\
\hline
\end{tabular}


Gráfico 1. Distribución de tipo de autoría según sexo de publicaciones en estudios de género en revistas indexadas en WOS

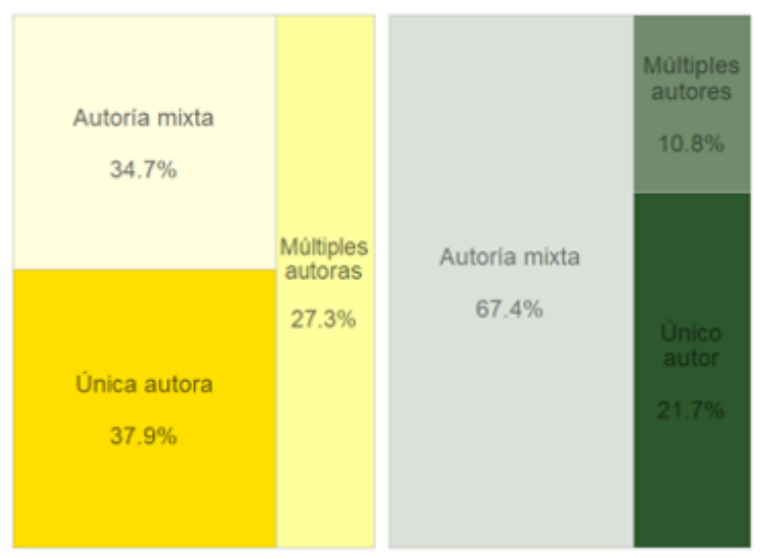

La tabla II presenta la cantidad de publicaciones por sexo, en ella se observa que la gran mayoría de las y los autores publicaron de forma ocasional (el $85,3 \%$ tuvo solamente una publicación, $84,1 \%$ en las autoras y $87,7 \%$ en los autores). Apenas un $1,9 \%$ de las y los autores tienen más de 3 publicaciones dentro del periodo (2,2\% para ellas y $1,4 \%$ para ellos), el autor que más publicó fue un hombre (18 artículos) y dentro de las y los 10 au- tores con más publicaciones hubo 6 mujeres y 4 hombres.

De momento, los datos presentados resumen la distribución y participación de las y los autores dentro del periodo. Sin embargo, no muestran información sobre la evolución temporal, cestos indicadores se mantuvieron constantes o hubo cambios a lo largo del periodo?

Para comenzar este análisis, la tabla III resume la cantidad de artículos y autoría por sexo en cada año. Allí se observa que en el año 2008 las publicaciones fueron 1.343 mientras que en 2018 fueron 2.698, dando cuenta de un crecimiento de $101 \%$.

En lo referente a las y los autores, el aumento fue algo superior $(112 \%)$, pasando de un total de 2.950 en 2008 a 6.243 en 2018 . Al segmentar por sexo se ve que las autoras crecieron un $115 \%$ y los autores $104 \%$. Para comprender mejor la relación, el gráfico 2 presenta el crecimiento porcentual de los cuatro segmentos.

En el gráfico 2 se muestra que el crecimiento de todos los segmentos fue similar, a medida que aumentó la cantidad de publicaciones lo hizo también la cantidad de las y los autores. Algunos aspectos que pueden destacarse es que el crecimiento no fue lineal, sino que tuvo tres

Tabla II. Distribución de las y los autores por cantidad de publicaciones

\begin{tabular}{|c|c|c|c|c|c|c|}
\hline Publicaciones & $\begin{array}{l}\text { Mujeres } \\
\text { autoras }\end{array}$ & $\begin{array}{c}\text { Hombres } \\
\text { autores }\end{array}$ & Total & $\begin{array}{l}\text { \% Mujeres } \\
\text { autoras }\end{array}$ & $\begin{array}{c}\% \text { Hombres } \\
\text { autores }\end{array}$ & \% Total \\
\hline 1 & 23.351 & 11.070 & 34.421 & $84,1 \%$ & $87,7 \%$ & $85,3 \%$ \\
\hline 2 & 2.973 & 1.119 & 4.092 & $10,7 \%$ & $8,9 \%$ & $10,1 \%$ \\
\hline 3 & 822 & 250 & 1.072 & $3,0 \%$ & $2,0 \%$ & $2,7 \%$ \\
\hline 4 & 315 & 88 & 403 & $1,1 \%$ & $0,7 \%$ & $1,0 \%$ \\
\hline 5 & 128 & 40 & 168 & $0,5 \%$ & $0,3 \%$ & $0,4 \%$ \\
\hline 6 & 74 & 26 & 100 & $0,3 \%$ & $0,2 \%$ & $0,2 \%$ \\
\hline 7 & 40 & 11 & 51 & $0,1 \%$ & $0,1 \%$ & $0,1 \%$ \\
\hline 8 & 18 & 6 & 24 & $0,1 \%$ & $0,0 \%$ & $0,1 \%$ \\
\hline 9 & 14 & 5 & 19 & $0,1 \%$ & $0,0 \%$ & $0,0 \%$ \\
\hline 10 & 5 & 0 & 5 & $0,0 \%$ & $0,0 \%$ & $0,0 \%$ \\
\hline 11 & 2 & 3 & 5 & $0,0 \%$ & $0,0 \%$ & $0,0 \%$ \\
\hline 12 & 5 & 1 & 6 & $0,0 \%$ & $0,0 \%$ & $0,0 \%$ \\
\hline 13 & 2 & 1 & 3 & $0,0 \%$ & $0,0 \%$ & $0,0 \%$ \\
\hline 14 & 2 & 1 & 3 & $0,0 \%$ & $0,0 \%$ & $0,0 \%$ \\
\hline 15 & 2 & 1 & 3 & $0,0 \%$ & $0,0 \%$ & $0,0 \%$ \\
\hline 16 & 0 & 0 & 0 & $0,0 \%$ & $0,0 \%$ & $0,0 \%$ \\
\hline 17 & 0 & 0 & 0 & $0,0 \%$ & $0,0 \%$ & $0,0 \%$ \\
\hline 18 & 0 & 1 & 1 & $0,0 \%$ & $0,0 \%$ & $0,0 \%$ \\
\hline Total & 27.753 & 12.623 & 40.376 & $100,0 \%$ & $100,0 \%$ & $100,0 \%$ \\
\hline
\end{tabular}


Tabla III. Distribución por año de publicaciones y autoría (por sexo) en estudios de género en revistas seleccionadas indexadas en WOS

\begin{tabular}{|c|c|c|c|c|c|c|c|}
\hline Año & $\begin{array}{c}\text { Total } \\
\text { artículos }\end{array}$ & $\begin{array}{c}\text { Total } \\
\text { autores }\end{array}$ & Autoras & Autores & $\begin{array}{c}\text { \% Mujeres } \\
\text { autoras }\end{array}$ & $\begin{array}{c}\text { \% Hombres } \\
\text { autores }\end{array}$ & $\begin{array}{c}\text { Cociente } \\
\text { Mujer/Hombre }\end{array}$ \\
\hline $\mathbf{2 0 0 8}$ & 1.343 & 2.950 & 2.020 & 930 & $68,5 \%$ & $31,5 \%$ & 2,17 \\
\hline $\mathbf{2 0 0 9}$ & 1.471 & 3.402 & 2.357 & 1.045 & $69,3 \%$ & $30,7 \%$ & 2,26 \\
\hline $\mathbf{2 0 1 0}$ & 1.581 & 3.510 & 2.460 & 1.050 & $70,1 \%$ & $29,9 \%$ & 2,34 \\
\hline $\mathbf{2 0 1 1}$ & 1.659 & 3.804 & 2.698 & 1.106 & $70,9 \%$ & $29,1 \%$ & 2,44 \\
\hline $\mathbf{2 0 1 2}$ & 1.594 & 3.644 & 2.575 & 1.069 & $70,7 \%$ & $29,3 \%$ & 2,41 \\
\hline $\mathbf{2 0 1 3}$ & 1.673 & 3.836 & 2.715 & 1.121 & $70,8 \%$ & $29,2 \%$ & 2,42 \\
\hline $\mathbf{2 0 1 4}$ & 1.747 & 3.955 & 2.793 & 1.162 & $70,6 \%$ & $29,4 \%$ & 2,40 \\
\hline $\mathbf{2 0 1 5}$ & 2.319 & 5.033 & 3.428 & 1.605 & $68,1 \%$ & $31,9 \%$ & 2,14 \\
\hline $\mathbf{2 0 1 6}$ & 2.550 & 5.572 & 3.931 & 1.641 & $70,5 \%$ & $29,5 \%$ & 2,40 \\
\hline $\mathbf{2 0 1 7}$ & 2.674 & 5.927 & 4.059 & 1.868 & $68,5 \%$ & $31,5 \%$ & 2,17 \\
\hline $\mathbf{2 0 1 8}$ & 2.698 & 6.243 & 4.343 & 1.900 & $69,6 \%$ & $30,4 \%$ & 2,29 \\
\hline Total & 21.309 & 40.376 & 27.753 & 12.623 & $68,7 \%$ & $31,3 \%$ & 2,20 \\
\hline
\end{tabular}

Gráfico 2. Crecimiento porcentual total de artículos, total de autores y autoría por sexo

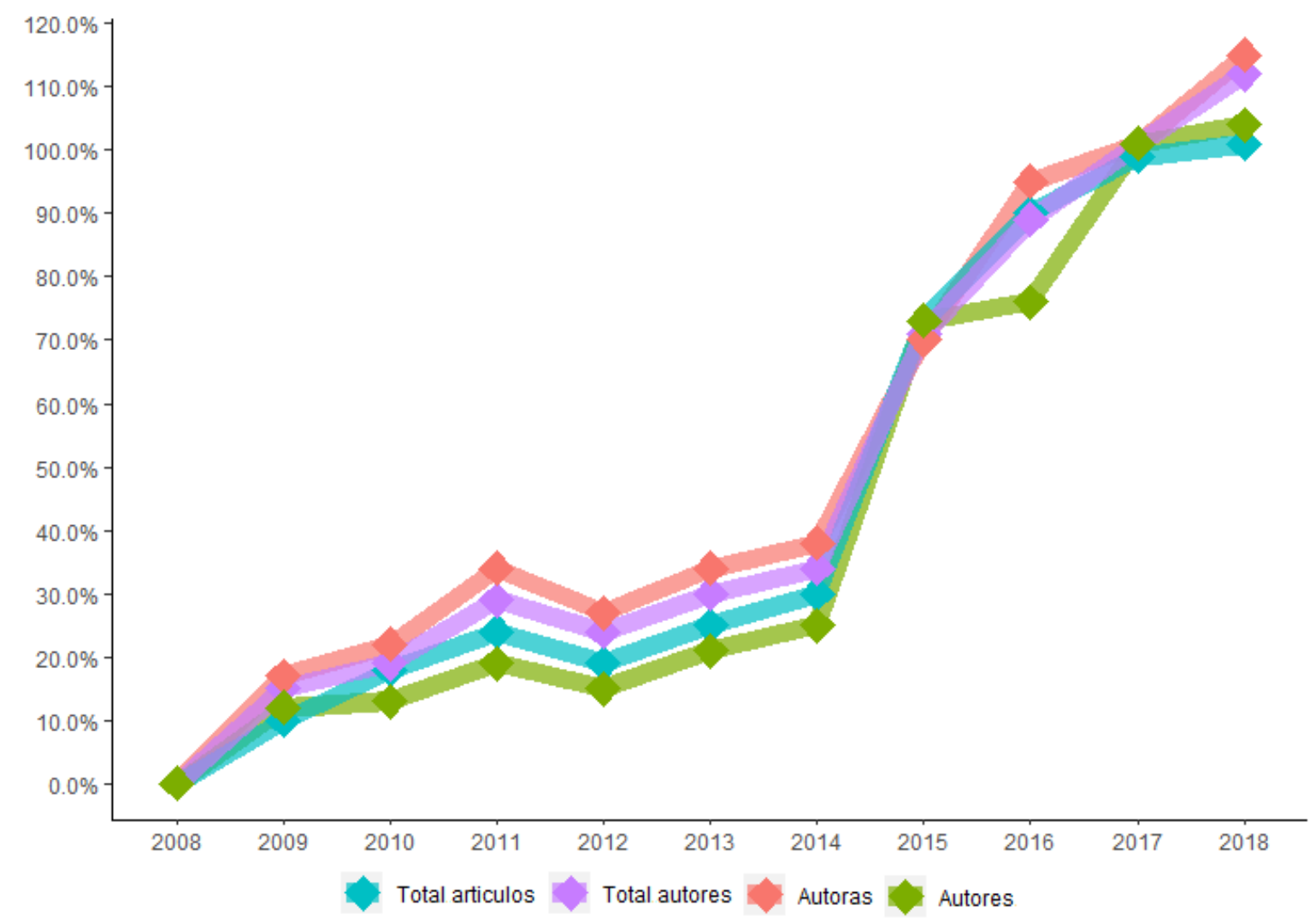


Gráfico 3. Distribución por tipo de autoría de publicaciones en estudios de género en revistas seleccionadas por año.

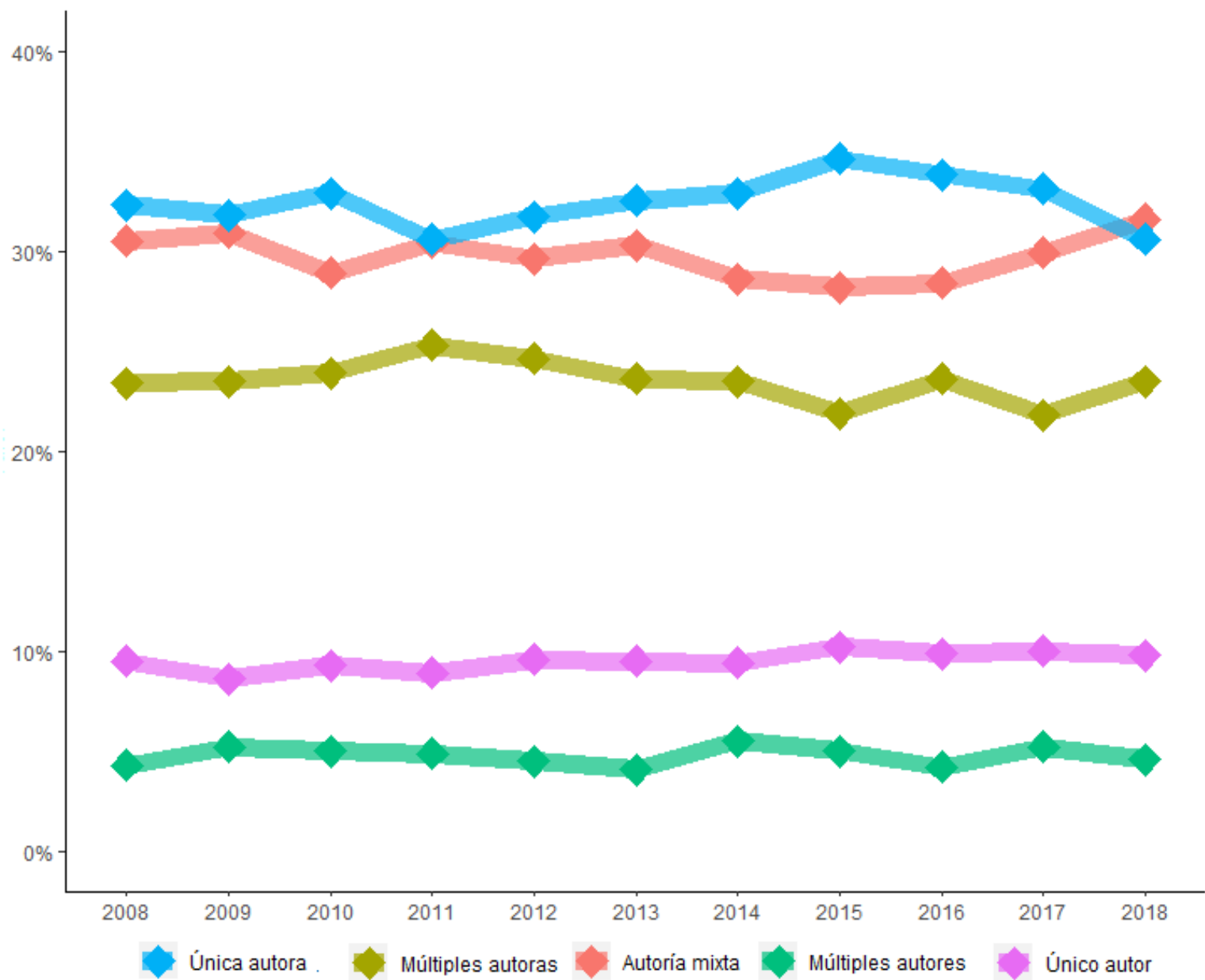

ciclos bien definidos: un crecimiento entre 2008 y 2011 , un estancamiento entre 2011 y 2014 y otro crecimiento (aún mayor) a partir de 2015. Debe aclararse que en este último año WOS incluye un nuevo índice (Emerging Sources Citation Index), lo que impacta en el fuerte incremento en ese año.

En la tabla III también se presenta la distribución porcentual de autoría por sexo y el cociente entre las y los autores. Al igual que los anteriores indicadores, ambos presentan una fuerte estabilidad a lo largo del período, la distribución anual por sexo fluctuó entre $68,1 \%$ y $70,9 \%$ para las autoras y entre $31,9 \%$ y $29,1 \%$ para los autores. El cociente entre autoras y autores lo hizo entre 2,42 y 2,14 mujeres por cada hombre. Finalmente, el gráfico 3 presenta la evolución temporal del tipo de autoría.

Como se observa en el gráfico 3, el tipo de autoría se mantuvo con pocas fluctuaciones durante el periodo. Sin embargo, a partir del año 2015 comienza a observarse la autoría mixta crece de manera constante, siendo el 2018 el primer año en que es la categoría con mayor porcentaje. Este fenómeno va en paralelo al descenso porcentual de las publicaciones con única autora, las que en 2018 (por primera vez) dejan de ser la categoría con mayor presencia. La participación en publicaciones por sexo (publicaciones donde participa al menos una autora, o un autor) se mantuvo sin grandes cambios, fluctuando entre $84,8 \%$ y $86,3 \%$ para las autoras y entre $42,6 \%$ y $45,9 \%$ para los autores, aunque debe destacarse que durante los últimos años presenta una tendencia similar a la de la autoría mixta (Gráfico 4).

Con respecto a la cantidad de artículos publicados por autor o autora, es un indicador que pierde importancia al hablar de un solo año ya que como se ha visto, el $85 \%$ de las y los autores tienen una solo publicación (ver tabla II). 
Gráfico 4. Participación por sexo en estudios de género en revistas seleccionadas por año.

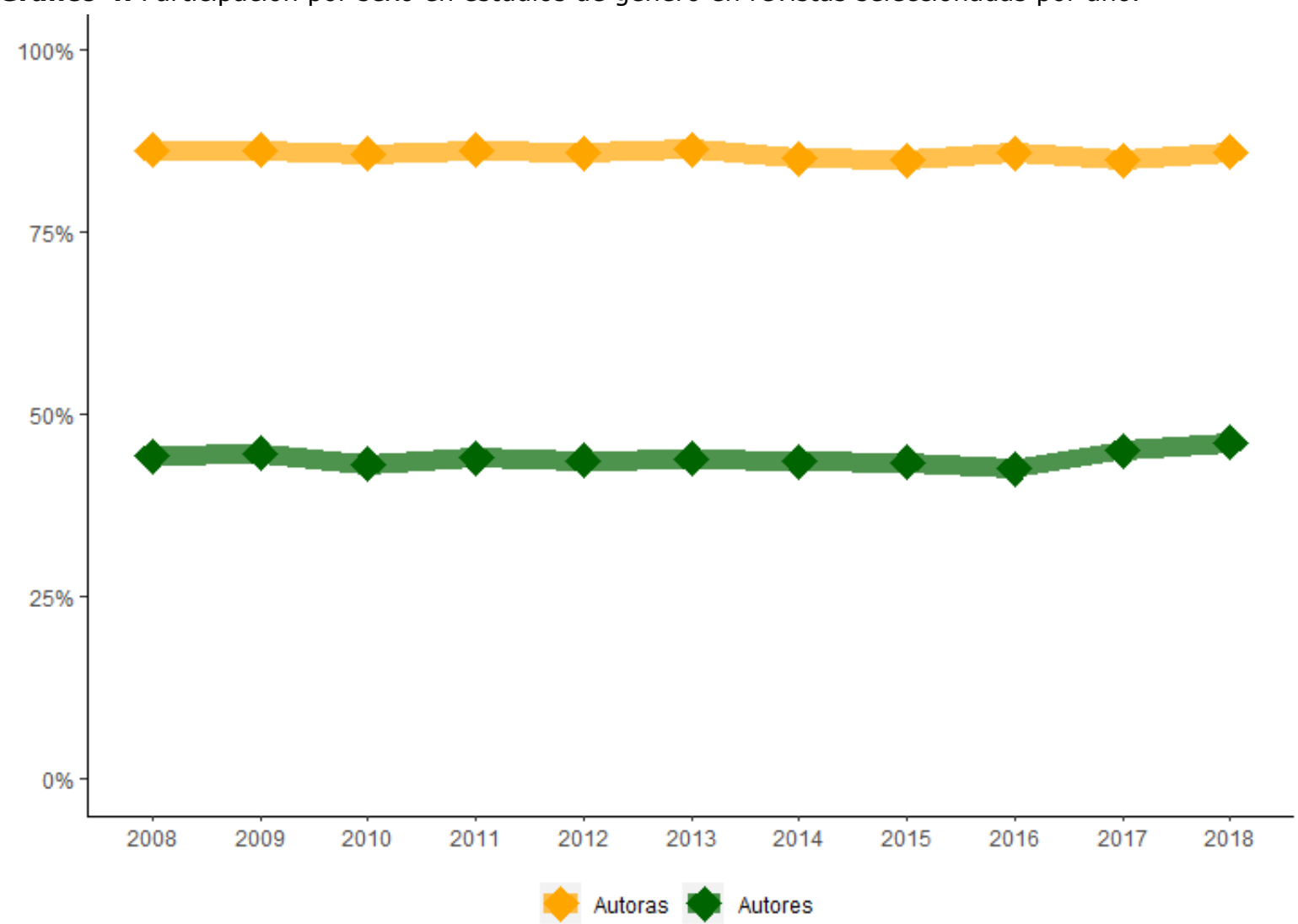

Fuente: elaboración propia en base a WOS.

\subsection{El contenido de las investigaciones}

Como se mencionó anteriormente, los estudios de género son un área transversal compuesta de una gran variedad de tópicos. Para identificarlos se aplicó $L D A$ y se obtuvieron 100 tópicos, los cuales se interpretaron a partir de las palabras con mayores probabilidades de aparición. Por ejemplo, el tópico 5 fue denominado "violencia de género" ya que las palabras más relevantes fueron: "violence", "victim", "ipv", "abuse"; en el tópico 99 las palabras más relevantes fueron "worker", "organization", "equality", "job", por lo tanto, se lo denominó "ámbito laboral".

La interpretación de los tópicos es un proceso manual, cualitativo, que requiere de la lectura de las palabras y su asociación a una temática. Existen tópicos fácilmente interpretables y otros que requieren bastante conocimiento del área para poder descubrirlos. Una de las características de este tipo de técnicas es que no toda la información es interpretable, existen tópicos difusos o incluso algunos presentan relaciones azarosas entre las palabras (Hall y otros, 2008). La tabla IV presenta los 38 tópicos identificados mediante la aplicación de $L D A$ y su distribución porcentual dentro del total de documentos (obtenida mediante el parámetro theta).

De acuerdo con la tabla IV, los tópicos identificados abarcan diversidad de temáticas: desde temas generales como medicina/salud (T23, T94, T48), economía (T8, T99), política (T62) a cuestiones específicas de los estudios del género como feminismo (T58), violencia de género (T5) y sexualidad en jóvenes (T11). También aparecen temas transversales de la literatura como salud reproductiva (T77) y otros más actuales como transgénero (T44). La gran variedad de temas permite reafirmar la transversalidad de los estudios de género a diferentes ámbitos y esferas sociales, políticas, culturales y del sistema científico.

La misma tabla IV también muestra la distribución porcentual de cada tópico (parámetro theta) y permite ver que no todos tuvieron igual probabilidad de aparición, algunos fueron más frecuentes (T58, T62) mientras que otros fueron mucho menos habituales (T84, T23). Generalmente, los tópicos de mayor probabilidad de aparición son 
Tabla IV. Tópicos identificados mediante LDA

\begin{tabular}{|c|c|c|c|c|c|}
\hline Tópico & Nombre & Dist \% & Tópico & Nombre & Dist $\%$ \\
\hline 1 & Abuso de sustancias & $0,9 \%$ & 43 & Cáncer de mama & $0,7 \%$ \\
\hline 4 & Violencia doméstica & $0,9 \%$ & 44 & Transgénero & $0,8 \%$ \\
\hline 5 & Violencia de género & $0,9 \%$ & 48 & HIV & $0,8 \%$ \\
\hline 6 & Embarazo & $1,2 \%$ & 54 & Masculinidad & $0,7 \%$ \\
\hline 8 & Brecha salarial & $1,0 \%$ & 56 & Salud pública & $0,9 \%$ \\
\hline 10 & Acoso sexual & $0,7 \%$ & 58 & Feminismo & $7,9 \%$ \\
\hline 11 & Sexualidad en jóvenes & $0,8 \%$ & 61 & Lenguaje & $0,7 \%$ \\
\hline 12 & Análisis cuantitativos & $1,7 \%$ & 62 & Movimiento político & $7,1 \%$ \\
\hline 14 & Trabajadoras sexuales & $0,6 \%$ & 65 & Leyes & $1,7 \%$ \\
\hline 16 & Imagen corporal & $1,4 \%$ & 67 & Estereotipos de género & $0,9 \%$ \\
\hline 20 & Racismo & $0,7 \%$ & 72 & Ensayos clínicos & $0,8 \%$ \\
\hline 23 & Salud mental & $0,7 \%$ & 77 & Salud reproductiva & $1,1 \%$ \\
\hline 25 & Sexismo & $0,7 \%$ & 80 & Ciudadanía & $0,9 \%$ \\
\hline 31 & Adolescencia & $1,0 \%$ & 84 & Etnias & $0,5 \%$ \\
\hline 35 & Satisfacción sexual & $0,9 \%$ & 88 & Estimaciones cuantitativas & $1,2 \%$ \\
\hline 36 & Discriminación & $0,8 \%$ & 91 & Análisis poblacionales & $1,8 \%$ \\
\hline 39 & Identidad género & $0,9 \%$ & 93 & Niñez & $1,1 \%$ \\
\hline 40 & Actividad física & $0,9 \%$ & 94 & Depresión & $2,0 \%$ \\
\hline 41 & Migraciones & $1,2 \%$ & 99 & Ámbito laboral & $1,5 \%$ \\
\hline
\end{tabular}

aquellos que definen conceptos, ideas y relaciones que pueden ser utilizadas dentro de un abanico de áreas temáticas. Los tópicos poco participativos, usualmente responden a temáticas locales / regionales, o a problemáticas no tan frecuentes.

Ahora bien, lo interesante del análisis no sólo radica en identificar estos temas, sino en descubrir una asociación entre los tópicos y el tipo de autoría, es decir, si las autoras y/o los autores se especializan en alguna temática en particular. Para ello se utilizó el puntaje de tópicos, indicador creado a partir de las distribuciones porcentuales de tópicos (obtenida mediante el parámetro theta).

La tabla V, además de presentar los puntajes, siguiendo los criterios definidos en la metodología, remarca en color rojo aquellos tópicos con un puntaje inferior a 0.75 y en verde a aquellos con uno superior a 1.25 , de esta forma resulta más claro identificar aquellos tópicos en donde se especializa (o no lo hace) cada uno de los tipos de autoría.

Lo primero a destacar es que tanto la especialización como la no especialización en tópicos se encuentra mucho más asociada al sexo de las y los autores que a la composición de la autoría (única o múltiple), por ello, para simplificar el análisis, se van a reducir los segmentos a 3: publicaciones de autoras (sin importar la cantidad), publicaciones de autores (sin importar cuantas sean) y publicaciones de autoría mixta.

En el caso de las autoras, se especializaron en 6 tópicos: violencia doméstica (T04), violencia de género (T05), imagen corporal (T16), feminismo (T58), movimiento político (T62) y leyes (T65). Asimismo, hubo 7 tópicos en los que no se especializaron, entre estos se pueden destacar varios asociados a estadística/metodología: análisis cuantitativos (T12), estimaciones cuantitativas (T88) y análisis poblacionales (T91).

En lo referente a los autores, no hubo tópicos con un puntaje mayor a 1.25 , lo que da cuenta de una producción más distribuida y no especializada. Sin embargo, existieron 9 tópicos por debajo de 0,75. Entre estos se pueden destacar varios asociados a medicina/salud en mujeres: embarazo (T06), cáncer de mama (T43), salud reproductiva (T77).

Por último, el tipo de autoría mixta se especializó en 9 tópicos, que pueden englobarse dentro de dos áreas temáticas: una asociada a la medicina/salud y otra a la estadística/metodología, por el contrario, no se especializaron en 3 tópicos, feminismo (T58), movimiento político (T62) y leyes (T65).

Más allá de las características de cada segmento, resulta relevante observar los comportamientos de 
Tabla V. Puntaje de tópicos según tipo de autoría.

\begin{tabular}{|c|c|c|c|c|c|}
\hline Tópico & $\begin{array}{c}\text { Única } \\
\text { autora }\end{array}$ & $\begin{array}{c}\text { Múltiples } \\
\text { autoras }\end{array}$ & $\begin{array}{c}\text { Autoría } \\
\text { mixta }\end{array}$ & $\begin{array}{l}\text { Múltiples } \\
\text { autores }\end{array}$ & $\begin{array}{l}\text { Único } \\
\text { autor }\end{array}$ \\
\hline T01 - Abuso de sustancias & 0.62 & 0.83 & 1.4 & 0.83 & 0.69 \\
\hline T04 - Violencia doméstica & 1.26 & 1.28 & 1.12 & 0.64 & 0.66 \\
\hline T05 - Violencia de género & 1.32 & 1.26 & 1.03 & 0.69 & 0.72 \\
\hline T06 - Embarazo & 0.93 & 1.07 & 1.13 & 0.74 & 0.73 \\
\hline T08 - Brecha salarial & 0.9 & 0.91 & 1.14 & 1.02 & 0.93 \\
\hline T10 - Acoso sexual & 1.08 & 1.06 & 0.89 & 1.14 & 1.13 \\
\hline T11 - Sexualidad en jóvenes & 1.02 & 0.98 & 0.91 & 1.13 & 1.14 \\
\hline T12 - Análisis cuantitativos & 0.47 & 0.64 & 1.61 & 1.14 & 0.95 \\
\hline T14 - Trabajadoras sexuales & 0.96 & 0.97 & 1.03 & 1.06 & 1.07 \\
\hline T16 - Imagen corporal & 1.26 & 1.21 & 0.75 & 1.01 & 1.1 \\
\hline T20 - Racismo & 0.94 & 0.99 & 1.07 & 0.89 & 0.92 \\
\hline T23 - Salud mental & 0.79 & 0.93 & 1.22 & 0.86 & 0.83 \\
\hline T25 - Sexismo & 0.84 & 0.89 & 1.11 & 0.99 & 0.9 \\
\hline T31 - Adolescencia & 0.86 & 0.9 & 1.15 & 1.1 & 0.95 \\
\hline T35 - Satisfacción sexual & 0.88 & 0.99 & 1.03 & 0.97 & 1 \\
\hline T36 - Discriminación & 1.11 & 1.05 & 0.9 & 1.02 & 1.07 \\
\hline T39 - Identidad género & 1.1 & 1.07 & 0.77 & 1.18 & 1.16 \\
\hline T40 - Actividad física & 0.6 & 0.81 & 1.42 & 0.86 & 0.68 \\
\hline T41 - Migraciones & 1.21 & 1.08 & 0.86 & 0.96 & 0.99 \\
\hline T43 - Cáncer de Mama & 0.82 & 0.96 & 1.32 & 0.73 & 0.64 \\
\hline T44 - Transgénero & 1.01 & 1.02 & 0.94 & 1.03 & 1.07 \\
\hline T48 - HIV & 0.81 & 0.95 & 1.11 & 0.95 & 0.96 \\
\hline T54 - Masculinidad & 0.9 & 0.97 & 1 & 1.09 & 1.12 \\
\hline T56 - Salud pública & 0.93 & 1.01 & 1.04 & 0.86 & 0.9 \\
\hline T58 - Feminismo & 1.34 & 1.26 & 0.7 & 1.06 & 1.11 \\
\hline T61 - Lenguaje & 1.11 & 1.05 & 0.89 & 1.04 & 1.11 \\
\hline T62 - Movimiento político & 1.32 & 1.25 & 0.69 & 1.04 & 1.12 \\
\hline T65 - Leyes & 1.31 & 1.16 & 0.72 & 1.06 & 1.09 \\
\hline T67 - Estereotipos de género & 0.81 & 0.94 & 1.11 & 1 & 0.93 \\
\hline T72 - Ensayos clínicos & 0.66 & 0.79 & 1.42 & 0.78 & 0.71 \\
\hline T77 - Salud reproductiva & 0.82 & 0.9 & 1.44 & 0.79 & 0.71 \\
\hline T80 - Ciudadanía & 1.22 & 1.18 & 0.79 & 1.1 & 1.12 \\
\hline T84 - Etnias & 0.99 & 1.02 & 1.06 & 0.85 & 0.82 \\
\hline T88 - Estimaciones cuantitativas & 0.48 & 0.73 & 1.56 & 0.92 & 0.89 \\
\hline T91 - Análisis poblacionales & 0.65 & 0.74 & 1.32 & 1.22 & 1.12 \\
\hline T93 - Niñez & 0.91 & 0.99 & 1.05 & 0.93 & 0.9 \\
\hline T94 - Depresión & 0.46 & 0.71 & 1.64 & 0.72 & 0.61 \\
\hline T99 - Ámbito laboral & 1.07 & 1.03 & 0.9 & 1.07 & 1.05 \\
\hline
\end{tabular}

forma comparada, identificar las similitudes y diferencias entre los tipos de autoría, trazando paralelos entre los tópicos en que se especializan y en los que no lo hacen.
Con respecto a las especializaciones, se observa que ninguno de los segmentos comparte tópicos, marcando una clara diferenciación entre lo que resulta relevante para cada grupo. En lo referente a 
Tabla VI. Resumen de especialización / no especialización de tópicos según segmento

\begin{tabular}{|l|l|l|l|}
\hline Tópico & Autoras & $\begin{array}{c}\text { Autoría } \\
\text { Mixta }\end{array}$ & Autores \\
\hline $\begin{array}{l}\text { T04 - Violencia } \\
\text { doméstica }\end{array}$ & & & \\
\hline $\begin{array}{l}\text { T05 - Violencia de } \\
\text { género }\end{array}$ & & & \\
\hline T58 - Feminismo & & & \\
\hline $\begin{array}{l}\text { T62 - Movimiento } \\
\text { político }\end{array}$ & & & \\
\hline T65 - Leyes & & & \\
\hline $\begin{array}{l}\text { T16 - Imagen } \\
\text { corporal }\end{array}$ & & & \\
\hline $\begin{array}{l}\text { T01 - Abuso de } \\
\text { sustancias }\end{array}$ & & & \\
\hline T40 - Actividad física & & & \\
\hline $\begin{array}{l}\text { T72 - Ensayos } \\
\text { clínicos }\end{array}$ & & & \\
\hline T94 - Depresión & & & \\
\hline $\begin{array}{l}\text { T12 - Análisis } \\
\text { cuantitativos }\end{array}$ & & & \\
\hline $\begin{array}{l}\text { T88 - Estimaciones } \\
\text { cuantitativas }\end{array}$ & & & \\
\hline $\begin{array}{l}\text { T91 - Análisis } \\
\text { poblacionales }\end{array}$ & & & \\
\hline $\begin{array}{l}\text { T43 - Cáncer de } \\
\text { mama }\end{array}$ & & & \\
\hline $\begin{array}{l}\text { T77 - Salud } \\
\text { Reproductiva }\end{array}$ & & \\
\hline T06 - Embarazo & & \\
\hline
\end{tabular}

la no especialización, de los 15 tópicos en los que no se especializa algún segmento, solamente hay 4 compartidos entre autoras y autores: abuso de sustancia (T01), actividad física (T40), ensayos clínicos (T72) y depresión (T94).

Lo más interesante de este análisis se encuentra en aquellos tópicos donde hay comportamientos opuestos, aquí podemos destacar 4 casos.

En primer lugar, los 4 tópicos en los que no se especializan ni las autoras ni los autores resultan tópicos relevantes para aquellas publicaciones de autoría mixta. En segundo lugar, los tópicos asociados a violencia de género (T04, T05) donde las autoras se especializan, no lo hacen los autores. En tercer lugar, en los tópicos asociados a estadística/metodología (T12, T88, T91) en los que se especializa la autoría mixta, no se especializan las autoras. Finalmente, los tópicos (T58, T62, T65) en los que se especializan las autoras, no son tópicos de especialización para la autoría mixta. La tabla VI resume la especialización / no especialización de tópicos según segmento.

\section{DISCUSIÓN}

Por medio de los resultados presentados se puede observar que la producción científica sobre estudios de género es un área feminizada. Los indicadores dan cuenta que hay más de 2 autoras por cada autor, un valor incluso superior al 1,86 encontrado en Thelwall y otros (2019); que la distribución de autoría por sexo (68,7\% de autoras) es mucho más alta que la presentada en Elsevier (2015; 2017; 2020) para las disciplinas con mayor presencia de mujeres y que supera ampliamente los márgenes de equilibrio de género ${ }^{4}$ propuestos por UNESCO (2019) y el Instituto Europeo de la Igualdad de Género (EIGE en sus siglas en inglés). Como plantea Penner (2015), los estereotipos culturales han inculcado que las mujeres tengan tendencia a participar en áreas asociadas al cuidado, la educación y las ciencias sociales más que en las áreas STEM, reforzando que las disciplinas históricamente feminizadas lo sigan siendo.

Al incluir la dimensión temporal, se observó un aumento de más $100 \%$ de las publicaciones y de las y los autores que participaron en publicaciones de los estudios de género en las revistas seleccionadas. Reafirmando el interés que los temas de estudio de esta disciplina han presentado en la década seleccionada.

Algo relevante de este punto es que no han existido cambios en las distribuciones por sexo dentro del periodo de estudio, esta característica ocurre también en otras áreas feminizadas como la enfermería (Elsevier, 2015, 2017, 2020) dando cuenta de una discordancia con lo que ocurre en gran parte de las áreas de investigación donde se observa un movimiento hacia la paridad de género (Elsevier 2015, 2017, 2020; Albornoz y otros, 2018).

Debe destacarse que a partir del año 2015 se observa una modificación en la composición de las publicaciones, dándose un aumento acotado, pero constante, en la autoría mixta, la que en el año 2018 supera por primera vez al porcentaje de la producción de las publicaciones con única autora. No hay evidencia suficiente, pero esto podría indicar un cambio de tendencia en la dinámica de publicación y por ende, que los equipos de trabajo y redes de investigación estén siendo constituidos cada vez más por personas de diferente sexo, lo que puede ser producto del advenimiento de los estudios de género como área de interés científico 
para ambos sexos (Núñez Noriega, 2016), como por políticas con perspectiva de género que promuevan la paridad en todos los ámbitos, incluyendo el de la investigación (UNESCO, 2017).

Dentro del contenido se ha encontrado que la cantidad de autores y autoras en las publicaciones no es una variable relevante, pero sí lo es la composición por sexo. Es decir que las investigaciones de una o varias mujeres tienen bastantes similitudes entre sí, lo mismo ocurre con las de uno o varios hombres y con aquellas con la autoría mixta.

Las autoras se especializan en violencia, teoría feminista, política, leyes, entre otras, y los autores por el contrario no se especializan en ningún tema. Los trabajos mixtos tienen una impronta de áreas asociadas a la medicina/salud y a la estadística/ metodología.

Dentro de los 38 tópicos identificados, los más relevantes han sido feminismo (T58) y movimiento político (T62), lo cual es consistente con lo expuesto por Bonder (2004) quien afirma que estos temas se encuentran dentro de los más trabajados en el campo de los estudios de la mujer y de género. Bonder (2004) también destaca otros temas como las cuestiones ligadas al acceso y permanencia en la educación, la legislación vigente, la comprensión de la violencia, tópicos que aparecen dentro de los encontrados (solo que no con tanta relevancia como plantea la autora).

Por otro lado, la evidencia encontrada no permite afirmar que la biomedicina como tal es el área más estudiada dentro las revistas de género, así como se plantea en Flores Espinola (2016), aunque sí queda claro que medicina/salud en general tiene una gran presencia y muchos de los tópicos identificados se vinculan a esta temática.

Los resultados tampoco permiten corroborar lo expuesto en Elsevier $(2015$; 2017) y Thelwall y otros (2019) de que los autores se asocian más a cuestiones vinculadas a la estadística/ metodología, aunque sí debe destacarse que estos conceptos se asocian a las publicaciones con autoría mixta y no se asocian a la autoría de mujeres.

\section{CONCLUSIONES}

El objetivo de la investigación consistió en responder a una serie de interrogantes asociados a la dinámica de la producción científica de los estudios de género en revistas seleccionadas de WOS.

Como se observó anteriormente, el interés por dichos estudios aumentó en el periodo de estudio, sin embargo, la participación de las y los autores en la producción científica se mantuvo constante siendo ellas las principales responsables de la producción en esta área de conocimiento, reafirmando a los estudios de género como un área feminizada. Esta misma feminización fue encontrada en los temas de investigación, algunos asociados a lo que eligen las mujeres: violencia, feminismo, política, leyes. La participación de los hombres es limitada y asimismo no presentan temas de especialización. Sin embargo, su aparición puede notarse cuando se trata de trabajos en conjunto, cuestión que se ha podido interpretar a la hora de identificar los trabajos de coautoría mixta y sus temas de especialización.

La utilización de algoritmos de clasificación por sexo y modelado de tópicos permitieron trabajar con grandes volúmenes de datos y obtener conclusiones más generalizables. El modelado de tópicos, además, permitió captar mejor el sentido de los documentos, ya que trabaja con todo el contenido del resumen y título, y no solamente con las palabras claves. Una ventaja que destacar de este tipo de estrategias es que, tras desarrollar los modelos, es posible extender el trabajo a otros años con gran facilidad.

La idea de analizar la dinámica de producción científica basada en la brecha de género, permite dar cuenta no sólo de la participación, sus cambios y tendencias, sino también de cuáles son los temas de trabajo que las y los autores eligen para abordar diferentes cuestiones que han permanecido en el tiempo, como los relacionados a la salud sexual y reproductiva de la sociedad, la violencia de género en todas sus formas o temas emergentes como la identidad de género, transexualidad, entre otros.

Es importante resaltar que los métodos utilizados en esta investigación aportan información actualizada para comprender la dinámica particular en la producción de los estudios de género, pudiendo ser aplicados a otras áreas y campos de conocimiento. Así entender cómo es la distribución de los sexos y la persistencia o no de la brecha de género en la ciencia.

En relación con esto, es posible interpretar cómo la academia sigue arrastrando consigo patrones culturales, estereotipos y construcciones colectivas sobre qué temas abordar de acuerdo con el sexo de las y los autores. Esto último es fundamental para la toma de decisión política, considerando que, ante la brecha de género en la participación e investigación de las mujeres en ciertos temas de importancia para la sociedad, el fomento a temáticas transversales, como las observadas en los resultados, no debería ser ajeno a ambos sexos. Esto podría dar lugar a transformaciones en el mundo científico y en las políticas públicas de la ciencia y 
de otros ámbitos de la sociedad como educación, salud, cultura, entre otros.

\section{AGRADECIMIENTOS}

Este artículo fue desarrollado en el marco de las actividades del Observatorio Iberoamericano de la Ciencia, la Tecnología y la Sociedad de la Organización de Estados Iberoamericanos para la Educación, la Ciencia y la Cultura (OCTS-OEI).

\section{ACKNOWLEDGEMENTS}

This paper was developed within the framework of the activities of the Ibero-American Observatory of Science, Technology and Society of the Organization of Ibero-American States for Education, Science and Culture (OCTS-OEI).

\section{NOTAS}

1 https://clarivate.com/webofsciencegroup/solutions/ web-of-science-core-collection. [08-02-2021]. 2 https://images.webofknowledge.com/images/help/wos/ hp research areas easca.html. [08-02-2021].

3 Las principales razones de no clasificación fueron la ausencia de nombre de pila (solo iniciales) o nombres muy poco frecuentes. De todos modos, fue posible identificar un $83 \%$ de los autores.

4 Equilibrio de género implica una representación igualitaria que en términos de distribución hace alusión a que dicha representación en cualquier órgano de toma de decisiones en la vida pública y política no debe ser inferior al $40 \%$

\section{REFERENCIAS}

Albornoz, M.; Barrere, R.; Matas, L.; Osorio, L.; Sokil, J. (2018). Las Brechas de Género en la Producción Científica Iberoamericana. En Red de Indicadores de Ciencia y Tecnología (RICYT) (coord.), El Estado de la Ciencia 2018, 31-46). http://www.ricyt.org/wp-content/uploads/2018/10/files_Estado-de-la-Ciencia2018_E_2018_BRECHAS_GENERO.pdf [Fecha de consulta: 08/02/2021].

Amorós, C.; de Miguel Alvarez, A. (2005). Teoría feminista, de la Ilustración a la globalización: Del feminismo liberal a la posmodernidad. Colección Estudios sobre la mujer. Minerva Ediciones.

Blei, D. M.; Ng, A.; Jordan, M. I. (2003). Latent Dirichlet Allocation. Journal of Machine Learning Research, 3, 993-1022. https://jmlr.org/papers/volume3/blei03a/ blei03a.pdf.

Bonder, G. (1984). Los Estudios de la Mujer y la Crítica Epistemológica a los Paradigmas de las Ciencias Humanas. Revista Desarrollo y Sociedad, 13, 27-38. https:// revistas.uniandes.edu.co/doi/10.13043/dys.13.1. https://doi.org/10.13043/dys.13.1

Bonder, G. (2004). Equidad de género en ciencia y tecnología en América Latina: bases y proyecciones en la construcción de conocimientos, agendas e institucionalidades. http://portal.oas.org/LinkClick.aspx?fileticket=mazNKYtWAVY\%3D\&amp;tabid=1527 [Fecha de consulta: 08/02/2021].
Elsevier (2015). Mapping Gender in the German Research Area. https://www.elsevier.com/research-intelligence/ resource-library/gender-2015 [Fecha de consulta: 08/02/2021]

Elsevier (2017). Gender in the Global Research Landscape. https://www.elsevier.com/research-intelligence/campaigns/gender-17 [Fecha de consulta: 08/02/2021].

Elsevier (2020). The Researcher Journey Through a Gender Lens. An examination of research participation, career progression and perceptions across the globe. https://www.elsevier.com/research-intelligence/resource-library/gender-report-2020 [Fecha de consulta: 08/02/2021]

Flores Espínola, A. (2016). ¿Los estudios CTS tienen un sexo? Mujeres y género en la investigación académica. Revista Iberoamericana de Ciencia, Tecnología y Sociedad, 31 (11). http://www. revistacts.net/contenido/numero-31/los-estudios-cts-tienen-un-sexo-mujeres-y-genero-en-la-investigacion-academica/.

Flores Espínola, A. (2010). Mujeres y feminismo en ciencia y tecnología: un análisis de revistas científicas. En Navarro, M; Estévez Cedeño, B; y Sánchez Cuervo, A. (eds.), Claves actuales de pensamiento, 171-194. Consejo Superior de Investigaciones Científicas (CSIC). https://editorial.csic.es/publicaciones/libros/11885/978-84-00-09255-9/claves-actuales-del-pensamiento-seminario-internac.html [Fecha de consulta: 08/02/2021].

González, M. I.; Fernández Jimeno, N. (2016). Ciencia, tecnología y género. Enfoques y problemas actuales. Revista Iberoamericana de Ciencia, Tecnología y Sociedad, 31 (11). https://www.redalyc.org/jatsRepo/924/92443623003/html/index.html.

Hall, D.; Jurafsky, D.; Manning, C. D. (2008). Studying the history of ideas using topic models. Proceedings of the Conference on Empirical Methods in Natural Language Processing, 363-371. https:// dl.acm.org/doi/10.5555/1613715.1613763 [Fecha de consulta: 08/02/2021] (Ponencia). https://doi. org/10.3115/1613715.1613763

Long, J. (1990). The Origins of Sex Differences in Science. Social Forces, 68 (4), 1297-1316. https://doi. org/10.2307/2579146.

López-Bassols, V.; Grazzi, M.; Guillard, C.; Salazar, M. (2018). Las brechas de género en ciencia, tecnología e innovación en América Latina y el Caribe: Resultados de una recolección piloto y propuesta metodológica para la medición. Banco Interamericano de Desarrollo (BID). http://dx.doi.org/10.18235/0001082 [Fecha de consulta: 08/02/2021].

Maffia, D. (2008). Carreras de obstáculos: las mujeres en ciencia y tecnología. En Actas del VII Congreso Iberoamericano de Ciencia, Tecnología y Género. Universidad de la Habana, http://www.ragcyt.org.ar/descargas/5202_doc.pdf [Fecha de consulta: 08/02/2021] (Ponencia).

Morales, S. (2018). ¿Qué está pasando con los estudios de género? Ciencia del Sur. https://cienciasdelsur. com/2018/10/29/que-esta-pasando-estudios-de-genero-escandalo/ (Blog).

Mullen, L. (2018). Gender: Predict Gender from Names Using Historical Data. R package version 0.5.2.https:// lincolnmullen.com/software/gender/ (Blog). 
Núñez Noriega, G. (2016). Los estudios de género de los hombres y las masculinidades: ¿qué son y qué estudian?. Culturales, 4(1), 9-31. http://www. scielo.org. $\mathrm{mx} /$ scielo.php? script $=\mathrm{sci}$ arttext\&pi$\mathrm{d}=\mathrm{S} 1870-11912016000100009 \& \operatorname{lng}=\mathrm{es} \& \mathrm{t} \operatorname{lng}=\mathrm{es}$.

Papadópulos, J.; Radakovich, R. (2006). Educación Superior y Género en América Latina y el Caribe. En UNESCO-IESALC (eds.), Informe sobre la educación superior en América Latina y el Caribe 2000-2005. La metamorfosis de la educación superior, 117-128. https://www.ses.unam.mx/curso2013/pdf/Papadopulos2006.pdf [Fecha de consulta: 08/02/2021].

Penner, A. M. (2015). Gender inequality in science. Science, 347 (6219), 234-235. https://doi. org/10.1126/science.aaa3781

Pérez Sedeño, E. (2001). A modo de introducción: las mujeres en el sistema de ciencia y tecnología. En Pérez Sedeño, E. (ed.). Las mujeres en el sistema de ciencia y tecnología. Estudios de casos, 9-17. OEI. https://geekgirlslatam.org/gg/wp-content/uploads/2020/09/mujeres-indigena-pdf.pdf [Fecha de consulta: 08/02/2021].

Thelwall, M.; Bailey, C.; Tobin, C.; Bradshaw, N. (2019). Gender Differences in Research Areas, Methods and
Topics: Can People and Thing Orientations Explain the Results?. Journal of Informetrics, 13(1), 149-169. https://doi.org/10.1016/j.joi.2018.12.002.

UNESCO. (2017). Plan de acción de la UNESCO, para la prioridad «Igualdad de Género». https://unesdoc. unesco.org/ark:/48223/pf0000227222_spa.

UNESCO. (2019). Women in Science. Fact Sheet, 55. http://uis.unesco.org/sites/default/files/documents/ fs55-women-in-science-2019-en.pdf (Informe).

Vogel, A.; Jurafsky, D. (2012). He Said, she Said: Gender in the ACL Anthology. En ACL-2012 Special Workshop on Rediscovering 50 Years of Discoveries, 33-41. https://www.aclweb.org/anthology/W12-3204/ [Fecha de consulta: 08/02/2021] (Ponencia).

Zuckerman, H.; y otros (1991). The Careers of Men and Women Scientists: A Review of Current Research. En Zuckerman, H.; Cole, J.; y Bruer, J. T. (eds.), The Outer Circle:-Women in the Scientific Community, 27-56. https://www.researchgate.net/ publication/232598611_The_Outer_Circle_Women_in_the Scientific_Community [Fecha de consulta: 08/02/2021] 